\title{
COMPARISON WITH OBSERVATIONS OF DISK GALAXIES
}

\author{
K. C. FREEMAN \\ Mount Stromlo and Siding Spring Observatory, Research School of Physical Sciences, \\ The Australian National University
}

\begin{abstract}
We discuss some observational aspects of the structure and dynamics of disk galaxies. Topics include the disk, the bulge, the lens, and the $z$-structure of the disk.
\end{abstract}

\section{Introduction}

First we recall the two-component (disk + bulge) structure of disk galaxies. Direct photographs and detailed surface photometry show how disk galaxies can have a very wide range in the relative importance of the disk and the bulge. For example, M33 and NGC 5907 have very weak bulges, NGC 4565 has a prominent but not dominant bulge, while the bulge of NGC 4594 is its dominant component. (Photographs of all these systems appear.in the Hubble Atlas.)

\section{The Disk}

The disk usually contributes a large part of the total light and angular momentum. For example, in M31 which has a fairly prominent bulge, the disk provides more than $75 \%$ of the total light, and probably more than $95 \%$ of the total angular momentum. Two comments about the angular momentum:

(1) We know that the disk's surface brightness distribution has the form $I(R)=$ $=I_{0} \exp (-\alpha R)$, and that for most disks $I_{0}$ is roughly constant at $21.65 \pm 0.3 \mathrm{~B}$ mag. $\operatorname{arcsec}^{-2}$, despite a 5 mag. range in total luminosity (Freeman, 1970). Now assume that the ratio of surface density to surface brightness $\mu(R) / I(R)$ is approximately constant within the disk: some support for this comes from the apparent uniformity of color for the disk and from Warner et al. (1973) observations of M33. Then $\mu(R)=$ $=\mu_{0} \exp (-\alpha R)$. Now the total luminosity $L=2 \pi I_{0} / \alpha^{2}$ and the total mass $M=2 \pi \mu_{0} / \alpha^{2}$ : because $M / L$ appears to be approximately constant for most spirals, it follows that $\mu_{0}$ is also approximately constant. The total angular momentum for an exponential disk in centrifugal equilibrium is $\mathscr{H}=1.1\left(G M^{3} / \alpha\right)^{1 / 2}$ so $\mathscr{H} \sim M^{7 / 4}$. This result follows dimensionally from the constancy of $\mu_{0}$, but is almost unverifiable observationally: see Freeman (1970) and Nordsieck (1973).

(2) Now consider the mass-angular momentum distribution $M(h)$, defined as the mass with angular momentum per unit mass less than $h$. A real disk will not be cold but will have at least enough velocity dispersion to stabilise it against local axisymmetric instabilities: i.e. $Q=\sigma / \sigma_{\min }>1$, where $\sigma_{\min }=3.36 \mathrm{G} \mu / \kappa$ is Toomre's (1964) minimum velocity dispersion for local axisymmetric stability and $\kappa$ is the local epicyclic frequency. Now a given $\mu(R)$ and $Q(R)$ distribution specifies $M(h)$ (although the con- 
verse is not true, at least in practice): i.e. if the disks have the exponential surface density distribution and have similar $Q(R)$ distributions, then they have similar $M(h)$ distributions. Small differences in the $Q(R)$ distribution do not change this conclusion significantly. For example, Kalnajs (personal communication) has constructed some selfconsistent disks with the 'isochrone' potential $\Phi=1 /\left(1+\sqrt{1+R^{2}}\right)$ : a $10 \%$ change in $Q(R)$ produces an almost imperceptible change in the $M(h)$ distribution. The origin of the $M(h)$ distribution is interesting for real galaxies. If the collapse to the disk was axisymmetric or only weakly barlike, then $M(h)$ was approximately invariant through the collapse and reflects the $M(h)$ for the protocloud, so we need to explain why the protoclouds had such similar $M(h)$ distributions. On the other hand, if the collapse was not axisymmetric, then we need to understand why $M(h)$ has in almost every system relaxed to this particular form.

\section{The Bulge}

This component has some features in common with elliptical galaxies. (1) Some bulges follow the $R^{1 / 4}$ law for their surface brightness distributions. (2) Spectrophotometry of the inner parts of some bulges shows that their spectra are very similar to those for the inner parts of some ellipticals, which in turn suggests that their stellar contents are similar. (3) This is reinforced by the apparent similarity of $M / L$ for the bulge of M31 and for normal ellipticals (see Emerson and Baldwin, 1973). (4) Some ellipticals show a change of integrated color with radius which probably reflects the same kind of radial population change as is well known for the bulge of the Milky Way. It seems likely that the bulge component is dynamically like an elliptical, but modified by rotation and the presence of the disk. No good theoretical account of the bulge dynamics is yet published: Carrick's work on this problem at Mt Stromlo will be available soon.

There is much interest now in invoking massive halos to stabilise disk galaxies against barlike deformation (see Ostriker and Peebles, 1973) and there is some indirect evidence that some spirals may have such halos. For example, the flat rotation curves observed for some spirals may imply large amounts of matter outside the visible disk: see M. S. Roberts' talk at this meeting. The total mass of each such spiral could then be of order $10^{12} M_{\odot}$, which would ease the present problem in binding gravitationally the groups of galaxies (Ostriker et al., 1974). It seems worth putting some observational constraints on such massive coronas. For example, say the halo has a mass of $10^{12} M_{\odot}$ and a radius of $300 \mathrm{kpc}$ : it is easy to show that this halo would have a significant effect on the dynamics of a typical disk. To make an estimate of the surface brightness of this halo, we can represent it by King's (1966) dynamical models, which give a realistic surface brightness representation for elliptical galaxies and globular clusters. We take the 'tidal' radius $r_{t}=300 \mathrm{kpc}$ and use two models: one with $\log \left(r_{t} / r_{c}\right)=2.23$ (concentrated, like an elliptical) and one with $\log \left(r_{t} / r_{c}\right)=1.39$ (less concentrated, like a globular cluster of intermediate concentration). The table below gives the projected surface density $\mu$ at $R=20 \mathrm{kpc}$ 
for the two models, and then the surface brightness at $R=20 \mathrm{kpc}$, assuming a $M / L_{B}$ of 200 for the halo.

\begin{tabular}{lll}
\hline $\log \left(r_{t} / r_{c}\right)$ & $\mu(20 \mathrm{kpc})$ & $I(20 \mathrm{kpc})$ \\
\hline 2.23 & $135 M_{\odot} \mathrm{pc}^{-2}$ & $27.5 \mathrm{Bmag} . \operatorname{arcsec}^{-2}$ \\
1.39 & 156 & 27.3 \\
\hline
\end{tabular}

Although this surface brightness is low, it is possible to test this picture observationally. For example, we have made detailed surface photometry of the nearby almost edge-on spiral NGC 253 , by averaging digitally several IIIa-J plates taken with a short focal length wide field Schmidt camera at Siding Spring. We can confidently exclude a surface brightness of $27.5 \mathrm{~B} \mathrm{mag}$. $\operatorname{arcsec}^{-2}$ at a height of $20 \mathrm{kpc}$ above the galactic plane in this system. Full details of this work will be published later. In summary, while it remains possible that spiral galaxies generally have massive halos with very large $M / L$ ratios, careful surface photometry can certainly put some harsh constraints on these halos.

In our Galaxy, the stellar population near the sun gives some information about the stellar content of the halo. Say the Galaxy, a typical large spiral, has a massive halo like those discussed above. In the solar neighborhood, a sphere of $10 \mathrm{pc}$ radius would then contain about $50 M_{\odot}$ of halo matter, which corresponds to at least several hundred faint stars, depending on the appropriate $M / L$. These stars would have typical velocities of 150 to $200 \mathrm{~km} \mathrm{~s}^{-1}$ (in each coordinate, relative to a nonrotating frame) for the halo to be in equilibrium. Now a velocity of $100 \mathrm{~km} \mathrm{~s}^{-1}$ at a distance of $10 \mathrm{pc}$ corresponds to a proper motion of $2 \operatorname{arcsec} \mathrm{yr}^{-1}$, but there do not appear to be any known stars with such large proper motions, parallaxes greater than 0 .'1 and intrinsically faint (say $M_{v}>15$ ). This may put a useful constraint on the stellar content of a massive halo, at least for the Galaxy.

A final point: we know that the halo of our Galaxy certainly extends to at least $100 \mathrm{kpc}$ radius. There are stars in the solar neighborhood with orbital apogalactica of $100 \mathrm{kpc}$ and more, and the globular clusters Pal 1, 3, 4, 13 and NGC 2419 are now about $100 \mathrm{kpc}$ from the galactic center. So it is not the large radial extent of the postulated halos that is unexpected, but rather the large associated mass.

\section{The Lens}

The disk itself often appears to have two components. This is particularly clear in $\mathrm{SO}_{1}$ lenticulars, where the disk is unconfused by spiral structure. Here is a quote from the Hubble Atlas: "To the eye, images of $\mathrm{SO}_{1}$ galaxies present three distinct luminosity zones on the original plate. There is an intense nucleus, an intermediate zone of lower surface brightness, called the lens, and the characteristic faint outer envelope." (This outer envelope is the exponential component in these systems.) Figure 1 shows the luminosity profile along the major axis of the SO system NGC 
1553. The lens, which can be seen on the short exposure photograph, also shows clearly as a plateau in the luminosity profile between about 1 and $2 \mathrm{kpc}$ from the nucleus. All three components described in the quote can be seen in the profile; in particular, Figure 1 demonstrates that the lens is not a photographic effect. The Hubble Atlas has many examples of systems with lenses: not only SOs but also spirals

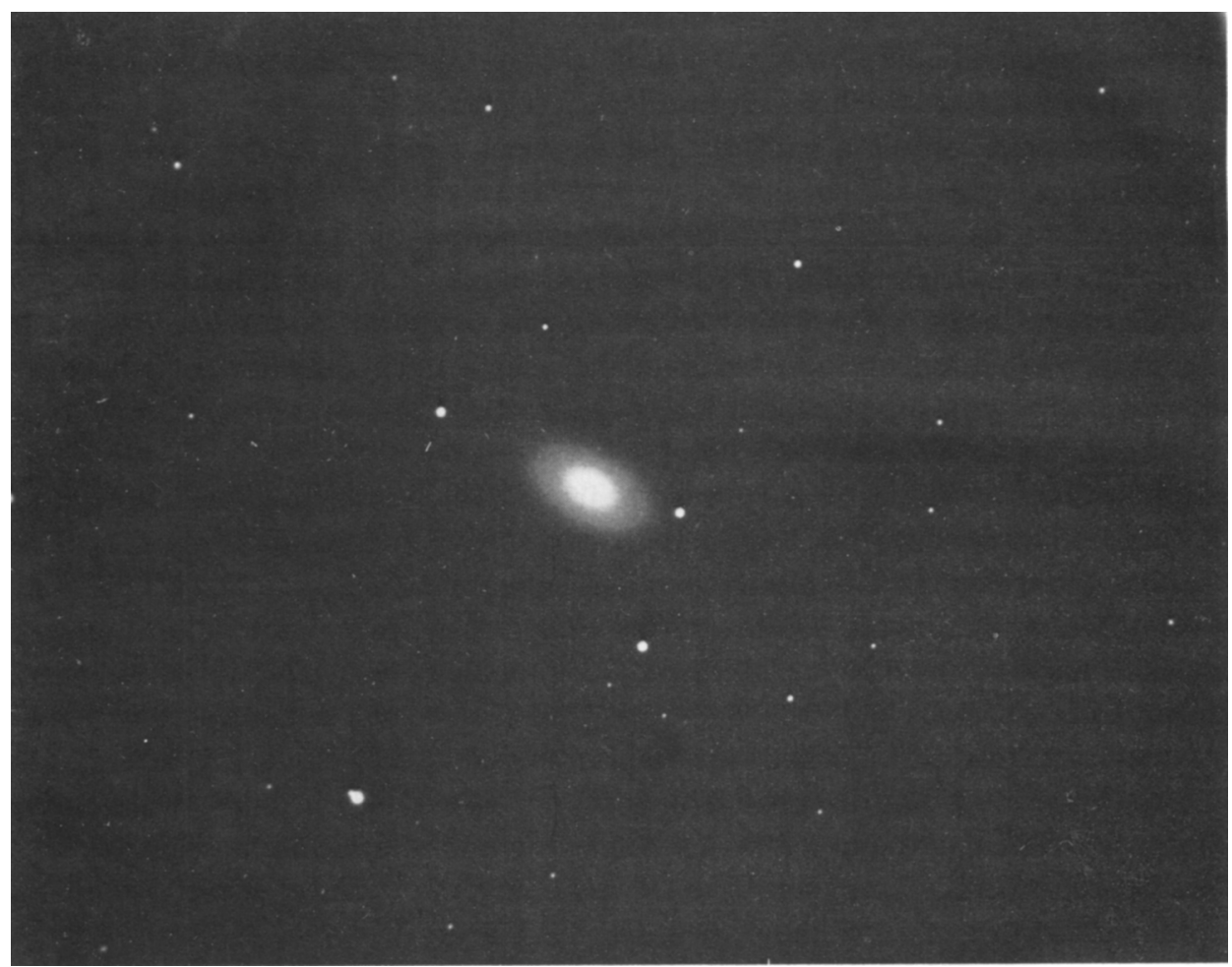

Fig. 1a. The SO galaxy NGC 1553. The nucleus and lens are visible.

like NGC 210 and NGC 1398 show a clear lens. So far, there seems to be no dynamical discussion of this component.

A few more facts: (1) In SBO systems the bar is very often immersed in the lens. See the Hubble Atlas. (2) Edge-on SOs, like NGC 4762 and NGC 7332, show that the lens is part of the flat disk component. (3) Not all disk galaxies have lenses. For example, there is no sign of a lens in the luminosity profile of M33 (de Vaucouleurs, 1959).

What is the lens dynamically? Maybe it is the hot inner disk that Ostriker and Peebles (1973) suggest as one way to stabilise the disk against barlike instabilities. However fact (1) above means that the lens is not always hot enough to do this effectively. Hohl's (1971) model disk evolution is interesting here: it shows the abrupt change in the gradient of the radial surface density distribution associated with a 


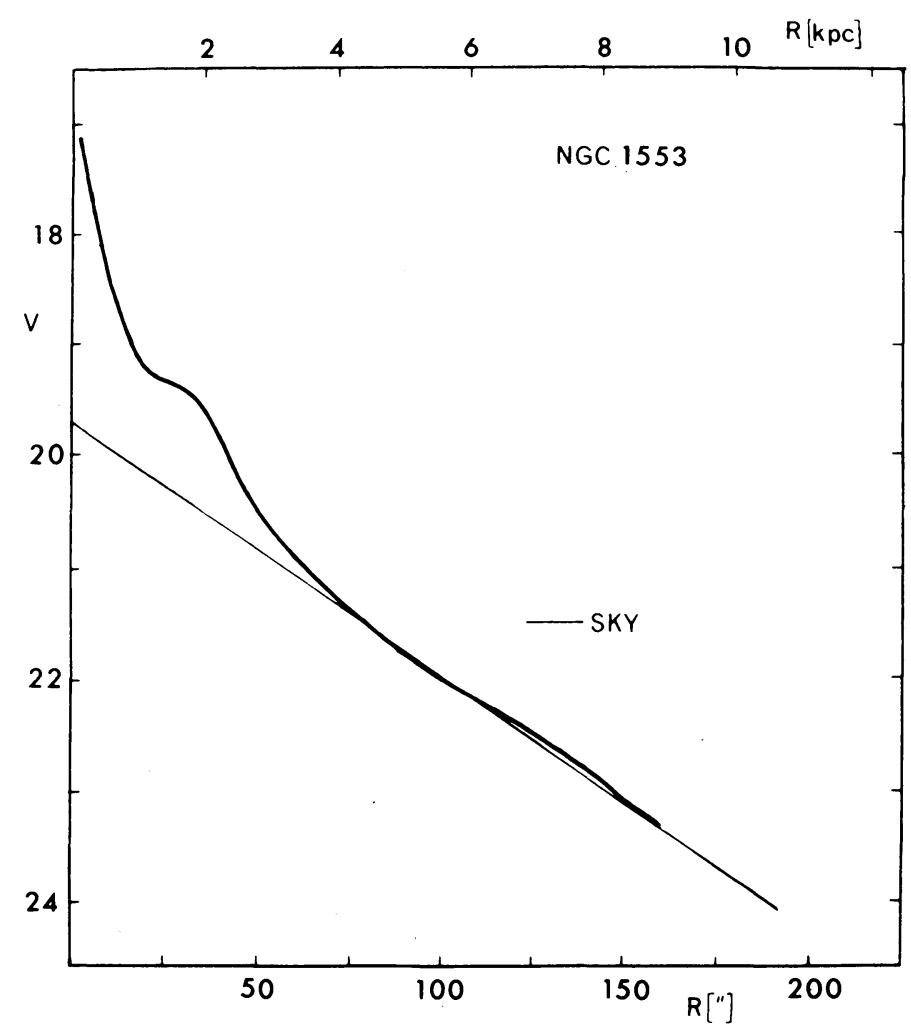

Fig. 1b. The luminosity profile for NGC 1553 (major axis). The surface brightness is in $V$ mag. $\operatorname{arcsec}^{-2}$. The lens appears as the plateau between 1 and $2 \mathrm{kpc}$.

change in the velocity dispersion gradient. It may be then that the lens is the hot inner disk, while the outer envelope may correspond to the cooler disk observed for our Galaxy in the solar neighborhood. This possibility can be tested observationally, at least in principle.

\section{The $z$-Structure of the Disk}

We know already how much can be learned about the formation and present structure of elliptical galaxies from their radial luminosity profile: this profile is associated with a particular distribution function (see I. R. King's talk), which itself probably originated through violent relaxation during the Galaxy's collapse phase. In the same way, there may be some information about the $z$-collapse of the disk, and its present $z$-structure, to be derived from studying the luminosity distribution in the $z$-direction for edge-on system, particularly as it seems likely that the $z$-dynamics of disk stars is almost decoupled from the dynamics in the plane. Edge-on SO galaxies are good candidates for this work, because the interstellar absorption in these systems is low. Mrs Grace is working on this problem at the University of Texas. 
Harvey Butcher and I have been studying the $z$-dependence of the integrated UBV colors in the disk of the edge-on SO NGC 4762. We find a clear change of $U-B$ with $z: \mathrm{U}-\mathrm{B}$ is smaller for larger $z$, and this could be analogous to the radial color variations in elliptical galaxies, if we believe that metal enrichment, occurring for the disk during its $z$-collapse, lead to a $z$-gradient of metal abundance in the disk.

\section{References}

Emerson, D. T. and Baldwin, J. E.: 1973, Monthly Notices Roy. Astron. Soc. 165, 9P.

Freeman, K. C.: 1970, Astrophys. J. 160, 811.

Hohl, F.: 1971, Astrophys. J. 168, 343.

King, I. R.: 1966, Astron. J. 71, 64.

Nordsieck, K.: 1973, Astrophys. J. 184, 719 and 735.

Ostriker, J. P. and Peebles, P. J. E.: 1973, Astrophys. J. 186, 467.

Ostriker, J. P., Peebles, P. J. E., and Yahil, A.: 1974, Astrophys. J. Letters 193, 1.

Toomre, A.: 1964, Astrophys. J. 139, 1217.

Warner, P. J., Wright, M. C. H., and Baldwin, J. E.: 1973, Monthly Notices Roy. Astron. Soc. $163,163$.

\section{DISCUSSION}

Brosche: With regard to the connection of mass and angular momentum it seems fallacious to condense the information of rotation curves into masses only and neglect the essentially two parameters which they provide (e.g. galaxies with a maximum rotational velocity of $1000 \mathrm{~km} \mathrm{~s}^{-1}$ could exist but are not observed and this has to be explained.)

King: Your remarks about false correlations between mass and angular momentum emphasize the importance of using observations only in the observational domain. To check a presumed relation between $M$ and $\mathscr{H}$, one should figure out what the consequence are for $R_{\text {char }}$ and $V_{\text {char }}$, and then look at those quantities. As regards your search for a halo in NGC 253, your model appears to drop off too rapidly to fit the rotation curve. If you had assumed a halo profile that corresponds to the rotation curve, you would expect even more brightness, and thus your negative observational result becomes even stronger.

Freeman: Agree with both comments.

Lecar: Do your observations exclude an Ostriker-Peebles halo which has a very flat surface density $(\sigma \sim 1 / r)$ ?

Freeman: One of the King models I used to estimate the expected surface brightness had $\log \left(R_{t} / R_{c}\right)=1.39$ which is a fairly unconcentrated model, and I think we can exclude this sort of distribution for $M / L=200$, $M \simeq 10^{12} M_{\odot}, R_{t}=300 \mathrm{kpc}$.

Innanen: A smooth empirical fit of the halo RR Lyrae star distribution to the local Population II data also does not appear to permit a massive halo component for the Galaxy. (See Innanen, K. A.: 1973, Astrophys. Space Sci. 22, 393).

Larson: If galactic halos are made of faint $M$ dwarfs, these stars radiate most of their energy at infrared wavelengths, and one should look for them at red or infrared wavelengths. In this case, I wonder how strong a limit you can set by looking only at blue wavelengths?

Freeman: The number I gave were for $M / L_{B}=200$. Going to the red has the advantages you mention but the sky brightness offsets some of this.

Miller: What do the radio astronomers have to say about these edge-on objects? Do any of them show structures like a halo?

Baldwin: In your disk and halo model of our Galaxy, the rotation curve will fit the observed one only if the core radius of the halo is comparable to $\alpha^{-1}$ in the disk. This occurs roughly half way between your models so they do indeed cover the right range of parameters. 